\title{
D-BAND (110-170 GHz) InP GUNN DEVICES
}

\author{
R. KAMOUA ${ }^{1}$, H. Eisele ${ }^{2}$ and G. I. HAdDad ${ }^{2}$ \\ 'Department of Electrical Engineering, State University of New York at Stony Brook, Stony Brook, \\ NY 11794-2350 and ${ }^{2}$ Center for Space Terahertz Technology, Department of Electrical Engineering and \\ Computer Science, The University of Michigan, Ann Arbor, MI 48109-2122, U.S.A.
}

(Received 14 December 1992)

\begin{abstract}
This paper reports on the development of InP Gunn sources for operation in the D-band $(110-170 \mathrm{GHz}) . n^{+}-n-n^{+}$structures with flat doping as well as graded doping profiles have been considered. Oscillations were obtained at $108.3 \mathrm{GHz}$ from a $1 \mu \mathrm{m}$ structure with a uniform $n$ doping of $2.5 \times 10^{16} \mathrm{~cm}^{-3}$. The CW RF output power was $33 \mathrm{~mW}$. A $1 \mu \mathrm{m}$ graded structure with an $n$ doping increasing linearly from $7.5 \times 10^{15}$ to $2.0 \times 10^{16} \mathrm{~cm}^{-3}$ has resulted in $20 \mathrm{~mW}$ at $120 \mathrm{GHz}$ and $10 \mathrm{~mW}$ at $136 \mathrm{GHz}$. These results are believed to correspond to a fundamental mode operation and represent the state-of-the-art performance from InP Gunn devices at these frequencies. This improvement in performance is attributed in part to a processing technique based on the use of etch-stop layers and InGaAs cap layers. An etch-stop layer allows low-profile mesas $(2-3 \mu \mathrm{m})$ and InGaAs cap layers help reduce the contact resistance, thus minimizing series resistances in the device. In addition, a physical model based on the Monte Carlo method was developed to aid in the design of structures for high frequency operation. Experimental results obtained from a $1.7 \mu \mathrm{m}$ Gunn device operating at W-band frequencies were used to estimate appropriate InP material parameters.
\end{abstract}

\section{INTRODUCTION}

Gunn devices are widely used as local oscillators up to $\mathrm{W}$-band $(75-110 \mathrm{GHz})$. At these frequencies the technology and circuit design are well developed. In particular at $94 \mathrm{GHz}, \mathrm{GaAs}$ and InP Gunn devices are available with high d.c. to RF conversion efficiencies and high RF power levels. The best performance obtained from GaAs Gunn devices is reported by Teng et al. [1]: $96 \mathrm{~mW}$ at $95 \mathrm{GHz}$ with $2.7 \%$ efficiency, $48 \mathrm{~mW}$ at $103 \mathrm{GHz}$ with $2.3 \%$ efficiency, and $34 \mathrm{~mW}$ at $110 \mathrm{GHz}$ with $1.6 \%$ efficiency. These results correspond to a second-harmonic mode of operation. Devices based on InP have shown higher power levels and oscillation frequencies compared to GaAs devices. The best performance to date from an $n^{+}-n-n^{+}$ structure has been reported by di Forte Poisson $e t$ al. [2], i.e. a maximum $\mathrm{CW}$ output power of $150 \mathrm{~mW}$ at $94 \mathrm{GHz}$ with an efficiency of $3.5 \%$. At higher frequencies the following experimental results have been reported: $30 \mathrm{~mW}$ at $97 \mathrm{GHz}[3], 35 \mathrm{~mW}$ at 105.8 $\mathrm{GHz}[4], 10 \mathrm{~mW}$ at $110 \mathrm{GHz}[5], 7 \mathrm{~mW}$ at $180 \mathrm{GHz}[7]$, and $0.2 \mathrm{~mW}$ at $272 \mathrm{GHz}[7]$. The results at 180 and $272 \mathrm{GHz}$ were obtained from InP Gunn devices operating in second and third harmonic modes, respectively. The results cited above can be further improved using current limiting contact structures [6], however the contacts are difficult to reproduce compared with contacts on $n^{+}$layers[8].

InP is recognized to have superior characteristics compared to GaAs for power generation in the millimeter wave region $[4,9]$. Fundamental mode operation up to $110 \mathrm{GHz}$ has been achieved with InP Gunn devices whereas GaAs Gunn devices are believed to operate in second harmonic mode at around $94 \mathrm{GHz}$ [10]. This paper reports on the development of InP Gunn devices in the D-band $(110-170 \mathrm{GHz})$. In particular the emphasis will be on developing fundamental oscillators. The approach adopted in this effort is both experimental and theoretical.

Experimentally, it was deemed important to improve the current processing technology. In particular, the various loss mechanisms need to be reduced. At the device level, the losses are due mainly to the skin effect and the contact resistance. Minimizing skin effect losses requires a mesa height less than $10 \mu \mathrm{m}$ [11] at frequencies above $100 \mathrm{GHz}$. The incorporation of etch-stop layers in the wafer design facilitates the complete elimination of the substrate and has been successfully employed in the fabrication of GaAs IMPact-Avalanche-Transit-Time (IMPATT) diodes $[12,13]$. An etch-stop layer consists of a thin epi-layer which reacts differently from the host semiconductor to various chemical etchants. With the advent of advanced material growth techniques (MOCVD, MBE, CBE, ..), such layers can be grown with excellent quality. In an InP system, InGaAs layers as etch-stop layers can be grown very close to the active region without compromising the crystal quality of the subsequent epi-layers. Optimizing the ohmic contacts to InP is increasingly important at high frequencies due to the reduced device area. As in the case of the etch-stop layer, a few thousand Angstrom InGaAs cap layer could be used to achieve this 
purpose. This layer is expected to improve the contact resistances as a result of the smaller bandgap of InGaAs compared with InP[14].

For the theoretical analysis, a physical model based on the Monte Carlo technique is developed to simulate, design, and predict the performance of Gunn structures for high frequency operation. A characteristic of the Monte Carlo method, as applied to the simulation of semiconductor devices, is the requirement of accurate values for a large number of material parameters. This is not an easy task, especially for the less technologically developed compounds such as InP. The typical material parameters given in the literature have been found to be inadequate in predicting our experimental data. Based on comparisons between the model and experimental results from a well characterized InP Gunn diode, more accurate material parameters were estimated.

The remainder of the paper is organized as follows: The next section presents an overview of the Monte Carlo model and describes the method used to evaluate the device performance. Section 3 describes the processing technique and highlights the various enhancements to the conventional integral heat sink method. Section 4 elaborates on the procedure used to determine the appropriate InP material parameters. The next section presents the experimental results obtained from a $1 \mu \mathrm{m}$ structure and the corresponding model predictions. Section 5 presents the experimental results and the theoretical predictions for a $1 \mu \mathrm{m}$ long structure with a graded doping profile. Finally Section 6 gives the summary and the conclusions.

\section{SIMULATION MODEL}

The self-consistent Ensemble Monte Carlo model is used to estimate the performance of InP Gunn devices at millimeter wave frequencies. This model is an extension of the one-particle Monte Carlo technique[15]. In order to describe the transport process in a Gunn structure where the doping is nonuniform and the fields are varying, an ensemble of electrons needs to be simulated simultaneously. The electric field has to be updated regularly since it evolves as the electrons redistribute in the structure. The analysis is carried out assuming the device behavior is mainly one dimensional which is justified for two terminal devices. A diode structure of length $L$ is divided into cells of equal length $(\Delta x=50 \AA)$. Any attributes of the electrons are averaged over each cell and assigned to the midway position of the cell. The cell size should be smaller than the smallest Debye length in the structure which occurs at the highly doped regions.

The simulation algorithm monitors the evolution in real space and momentum space of an ensemble of electrons. The simulation time is partitioned into time steps ( $\Delta t=5 \times 10^{-15} \mathrm{~s}$ ), and each time step is terminated by a call to a Poisson equation solver in order to update the electric field. In each time step, every electron is submitted to successive free flights terminated by a scattering process which is selected using a random number generator. Electrons crossing cell boundaries are temporarily stopped at that boundary and then resumed with the electric field in the new cell. An analogous procedure is followed when it is time to update the electric field and the electron is in the middle of a free flight. In this case the remaining flight time is stored, and the flight is resumed after all other electrons are simulated for one time step, the carrier density is calculated, and the electric field is updated.

To predict the performance of a particular Gunn structure a sinusoidal RF voltage is applied across the device and the current response is simulated over many RF periods (about 10). A Fourier analysis of the resulting particle current density gives the current's fundamental component which is subsequently used to determine the device admittance per unit area. To estimate the RF output power, the Gunn device is assumed to be connected to a resonant circuit represented by a load resistance and a resonating inductance. A series resistance is included in the equivalent circuit to take into account effects of contact resistances, any substrate resistances, and skin effect losses.

\section{InP GUNN TECHNOLOGY}

The most common fabrication techniques for Gunn devices are based on the Integral Heat Sink process (IHS) [16,17] or the flip-chip process[18]. In the IHS process, the heat sink is formed as an integral part of the diode. The wafer front side is metallized and plated with copper, silver, or gold to a thickness of several thousanths of an inch. The substrate is then chemically or mechanically thinned to a thickness of $10-15 \mu \mathrm{m}$. Next, ohmic contacts are formed and standard photoresist techniques are used to define the mesas. Individual chips are then mounted in standard packages. In the flip-chip process the mesas are defined on the epitaxial side. The mesa chip is then flipped and mounted on a heat sink pedestal. Finally, the substrate is thinned to about $100 \mu \mathrm{m}$.

Both processing techniques have been successfully used to fabricate Gunn devices operating at W-band frequencies. At still higher frequencies, it is necessary to further reduce the substrate thickness in order to minimize the series resistance. In addition, the smaller size of the mesas decreases the yield in the packaging and bonding procedure. Therefore a fabrication technology has been developed that allows the complete removal of the substrate. This process is a modification and extension of the above mentioned IHS process and includes the additional step of plating the top contacts to facilitate bonding.

Figure 1 shows the layer sequence of the InP-based epitaxial material. The different epitaxial layers are grown on an $n^{+}$doped InP substrate. They consist of a highly doped InGaAs etch-stop layer, a highly 


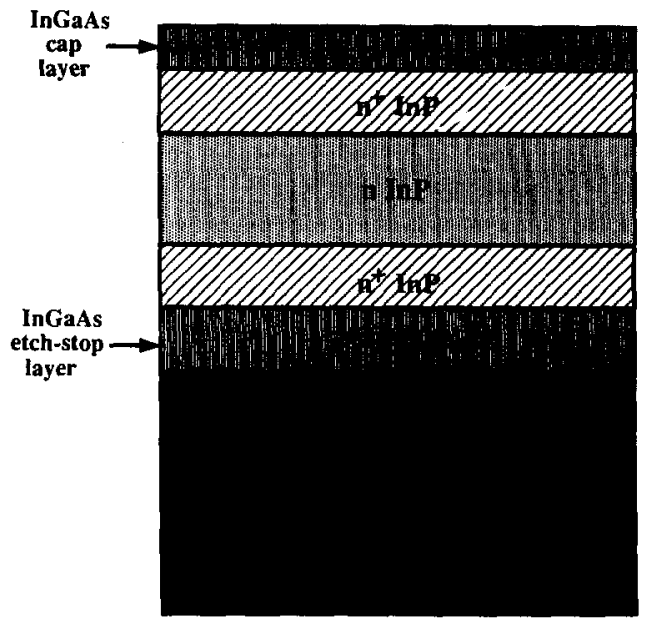

Fig. 1. Layers of a typical InP Gunn wafer.

doped InP contact layer, a moderately doped InP active region, a highly doped top InP contact layer, and a highly doped InGaAs cap layer. Prior to processing the wafer, a small sample piece is cleaved off. The highly $n$ doped top layers are etched away, Schottky contacts are formed on the active region, and the doping profile in the active region is determined by $\mathrm{C}-V$ measurements. The important steps in the processing sequence are shown in Fig. 2.

In the first step square islands of approximately $400 \times 400 \mu \mathrm{m}$ are defined and separated from each other by $100 \mu \mathrm{m}$ wide trenches. These trenches are etched down to the InGaAs etch-stop layer. An $n$-ohmic contact $(\mathrm{Ni} / \mathrm{Ge} / \mathrm{Au} / \mathrm{Ti} / \mathrm{Au})$ is evaporated over the whole surface and then gold or silver is plated to a thickness between 15 and $25 \mu \mathrm{m}$ to form the integral heat sink. The isolation provided by the trenches effectively reduces the formation of cracks in the epi-layers during the annealing process. Cracks are caused by the difference in the thermal expansion coefficient between the semiconductor and the heat sink. In addition, the trenches keep the sample flat which facilitates further processing and handling. Figure 2(a) shows a cross section of the sample after plating of the heat sink.

After forming the heat sink, the substrate is thinned and the top contacts are defined. A hydrochloric acid based solution ( $\mathrm{HCl}: \mathrm{H}_{2} \mathrm{O} 4: 1$ ) selectively etches the substrate away and stops at the InGaAs etch-stop layer. Once the substrate is removed, the InGaAs is either etched away or left intact to be used as a cap layer. Standard lithography techniques are used to define circular diodes with sizes varying from 30 to $65 \mu \mathrm{m}$ in diameter which are then metallized to form $n$-ohmic contacts. Figure 2(b) shows a cross section of the sample after substrate thinning and contact definition.

In the standard IHS technique, the next step would be to etch the mesas. However bonding problems have been encountered whenever thin evaporated metal layers are used for the ohmic contacts. One solution can be found in plating gold on top of the evaporated layers. This results in an additional alignment step and a longer processing time. The complete procedure consists of evaporating a conductive metal multilayer $(\mathrm{Ti} / \mathrm{Au} / \mathrm{Ti})$ over the sample. The sample is then coated with a thick photoresist layer $(3 \mu \mathrm{m})$. Standard lithography techniques are used to remove the photoresist from selective areas where the ohmic contacts are formed. The $\mathrm{Ti}$ layer in the exposed area is removed and the sample is plated to a thickness of $2-3 \mu \mathrm{m}$. Figure $2(\mathrm{c})$ shows a cross section of the sample after plating.

In the final step prior to mounting individual devices, the semiconductor material between the circular contacts is etched away to form the mesa. A chemical solution based on hydrochloric acid and phosphoric acid $\left(\mathrm{HCl}: \mathrm{H}_{3} \mathrm{PO}_{4} \quad 1: 3\right)$ is used for this etch. The sample is subsequently annealed on a hot

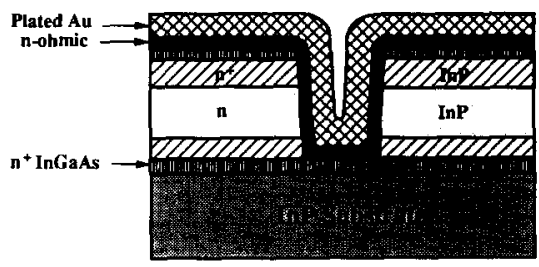

a) Island definition, n-ohmic evaporation, and gold plating $(25 \mu \mathrm{m})$

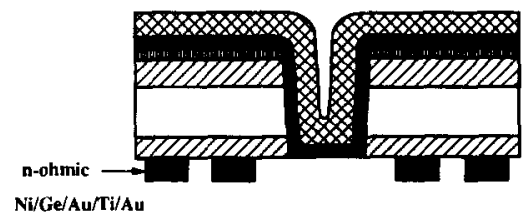

b) Substrate thinning, etch stop layer removal, and n-ohmic evaporation

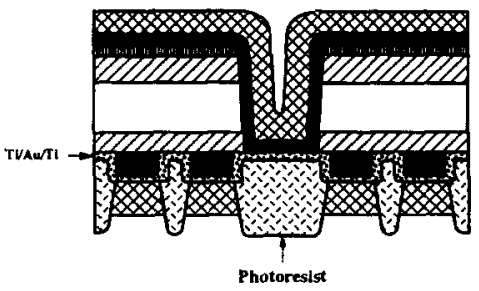

c) Gold plating of ohmic contacts

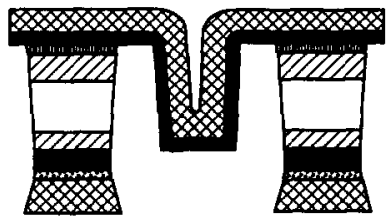

d) Diodes after annealing and mesa etch

Fig. 2. Processing sequence for InP Gunn device fabrication. 
plate at various temperatures $\left(380-405^{\circ} \mathrm{C}\right)$. A cross section of the final structure is shown in Fig. 2(d).

\section{ESTIMATION OF InP MATERIAL PARAMETERS}

In Section 2, a Monte Carlo model for simulating the performance of Gunn structures was described. In this section, the material parameters needed for the Monte Carlo model are estimated. The accuracy of the model is strongly dependent on the accuracy of the material parameters used. Unfortunately, a wide range of values is given in the literature. In particular, some of the material parameters that are important to the Gunn effect have the following range of values[19-23]:

\section{$\Gamma-L$ valley separation $(\mathrm{eV})$}

$0.4 \leftrightarrow 0.832$,

$L$ valley effective mass ratio $\left(\mathrm{M} / \mathrm{M}_{0}\right) \quad 0.26 \leftrightarrow 0.4$,

$\Gamma-L$ coupling constant $\left(\times 10^{9} \mathrm{eV} / \mathrm{cm}\right) \quad 0.1 \leftrightarrow 2.5$,

$\Gamma-X$ coupling constant $\left(\times 10^{9} \mathrm{eV} / \mathrm{cm}\right) \quad 0.43 \leftrightarrow 1.0$.

There is more than an order of magnitude uncertainty in the $\Gamma$ to $L$ intervalley coupling constant. The appropriate material parameters are determined by comparing measurements at high frequencies with results predicted by the model. The structure considered for comparison is shown in Fig. 3. It has a $1.7 \mu \mathrm{m}$ long active region doped at $1 \times 10^{16} \mathrm{~cm}^{-3}$, a $0.1 \mu \mathrm{m}$ cathode region doped at $3 \times 10^{17} \mathrm{~cm}^{-3}$, and a $0.2 \mu \mathrm{m}$ anode region doped at $3 \times 10^{17} \mathrm{~cm}^{-3}$.

An InP wafer with this structure has been processed using the technology described above. Diodes with various sizes have been mounted on copper heat sinks. Tapered leads were thermocompression bonded to the diode and to four metallized quartz standoffs. For some diodes, a metallized quartz ring was used instead of the standoffs.

A $50 \mu \mathrm{m}$ diode was tested in a W-band resonant cavity with the following results:

$\begin{array}{ll}\text { bias voltage } & 5.0 \mathrm{~V}, \\ \text { oscillation frequency } & 80.0 \mathrm{GHz}, \\ \text { output power } & 40.0 \mathrm{~mW}, \\ \text { conversion efficiency } & 1.6 \% \text {, and } \\ \text { d.c. current } & 500 \mathrm{~mA} .\end{array}$

\begin{tabular}{|c|c|c|}
\hline$n^{+} \operatorname{InP}$ & $\mathrm{n} \operatorname{InP}$ & $\mathrm{n}^{+} \operatorname{InP}$ \\
$3 \times 10^{17} \mathrm{~cm}^{-3}$ & $1 \times 10^{16} \mathrm{~cm}^{-3}$ & $3 \times 10^{17} \mathrm{~cm}^{-3}$ \\
\hline
\end{tabular}

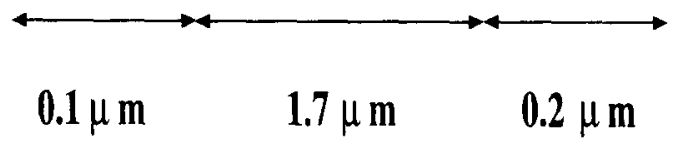

Fig. 3. Gunn structure used in the model to determine more accurate InP material parameters.
Table 1. InP initial material parameter set

\begin{tabular}{lcl}
\hline Energy separation (eV) & $\Gamma-L$ & 0.832 \\
& $\Gamma-X$ & 1.5 \\
Effective mass $\left(\frac{m}{m_{0}}\right)$ & $\Gamma$ & 0.082 \\
& $L$ & 0.26 \\
Nonparabolicity factor (1/eV) & $X$ & 0.325 \\
& $\Gamma$ & 0.83 \\
& $L$ & 0.23 \\
Intervalley coupling constant & $X$ & 0.38 \\
(109 eV/cm) & $\Gamma-L$ & 0.506 \\
& $\Gamma-X$ & 0.498 \\
& $L-X$ & 0.468 \\
Acoustic deformation potential (eV) & $L-L$ & 0.575 \\
& $X-X$ & 0.28 \\
& $\Gamma$ & 7 \\
LO phonon energy (eV) & $X$ & 7 \\
& $X$ & 7 \\
Static dielectric constant & $\Gamma$ & 0.043 \\
Optical dielectric constant & $L$ & 0.0423 \\
\hline
\end{tabular}

The structure shown in Fig. 3 is simulated using the Monte Carlo model. The d.c. bias voltage is set to $5.0 \mathrm{~V}$ and the temperature to $450 \mathrm{~K}$. A starting set of material parameters is assumed and is taken from various sources in the literature. These parameters are listed in Table 1 and constitute the initial parameter set. No oscillations were obtained with these values for frequencies ranging from 75 to $120 \mathrm{GHz}$. It appears that the $\Gamma-L$ intervalley energy separation of $0.8 \mathrm{eV}$ is too large. A systematic procedure for changing the values of the different parameters is adopted. In particular the values used for the intervalley energy separation and the effective mass in each valley are targeted. It is expected that the occurrence of oscillations will be enhanced if the electron effective mass in the satellite valleys is increased, the intervalley energy separation is reduced, and the scattering rates to the satellite valleys are increased. The combined effect of these changes is to increase the transfer to the satellite valleys for the same bias

Table 2. InP final material parameter set

\begin{tabular}{lll}
\hline Energy separation (eV) & $\Gamma-L$ & 0.45 \\
& $\Gamma-X$ & 0.775 \\
Effective mass $\left(\frac{m}{m_{0}}\right)$ & $\Gamma$ & 0.082 \\
& $L$ & 0.5 \\
Nonparabolicity factor (1/eV) & $X$ & 0.5 \\
& $\Gamma$ & 0.83 \\
& $L$ & 0.23 \\
Intervalley coupling constant & $X$ & 0.38 \\
(10 $0^{9}$ eV/cm) & $\Gamma-L$ & 1.0 \\
& $\Gamma-X$ & 1.0 \\
& $L-X$ & 0.468 \\
Acoustic deformation potential (eV) & $L-L$ & 0.575 \\
& $X-X$ & 0.28 \\
& $\Gamma$ & 5 \\
LO phonon energy (eV) & $X$ & 5 \\
& $\Gamma$ & 5 \\
Static dielectric constant & $L$ & 0.043 \\
Optical dielectric constant & $X$ & 0.0423 \\
\hline
\end{tabular}




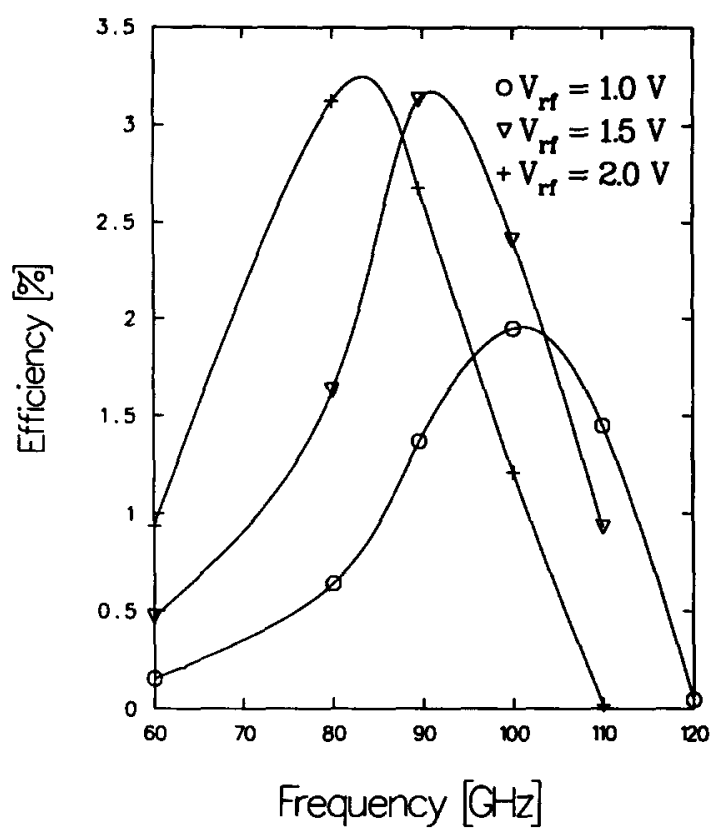

Fig. 4. Predicted efficiency as a function of frequency for three RF voltages $(1.0,1.5$ and $2.0 \mathrm{~V})$. The d.c. voltage is $5.0 \mathrm{~V}$ and the operating temperature is $450 \mathrm{~K}$.

voltage and reduce the average electron velocity at high electric fields. As a consequence a larger negative differential mobility is obtained which is more favorable for nucleating space charge layers. Upon making the above changes a set of parameters, listed in Table 2, was obtained which will be shown to yield good agreement with the experimental results.

In order to compare the theoretical predictions with the experimental results, the diameter of the

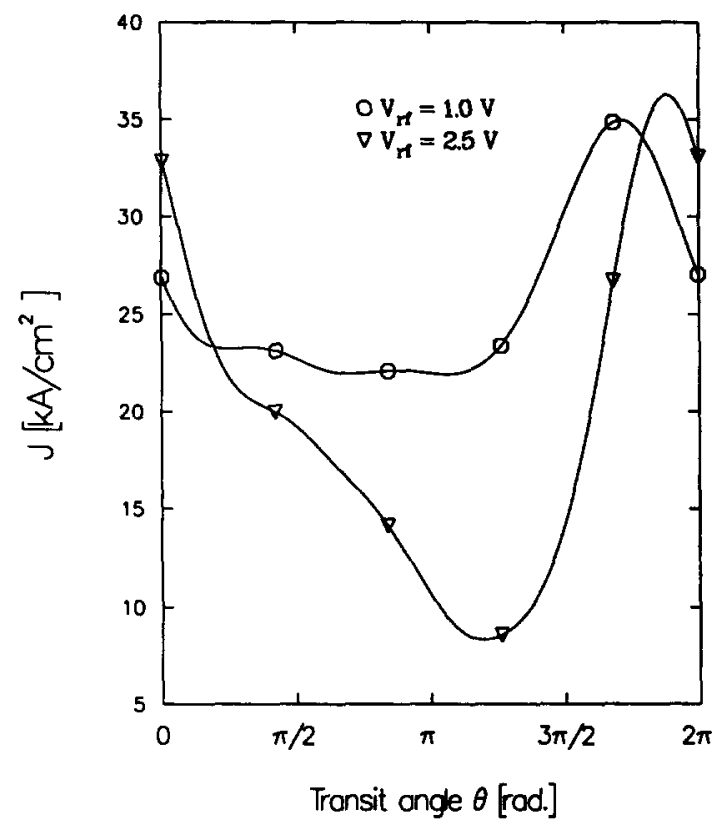

Fig. 5. Comparison of the current density response at $105 \mathrm{GHz}$ for two RF voltages: 1.0 and $2.0 \mathrm{~V}$.

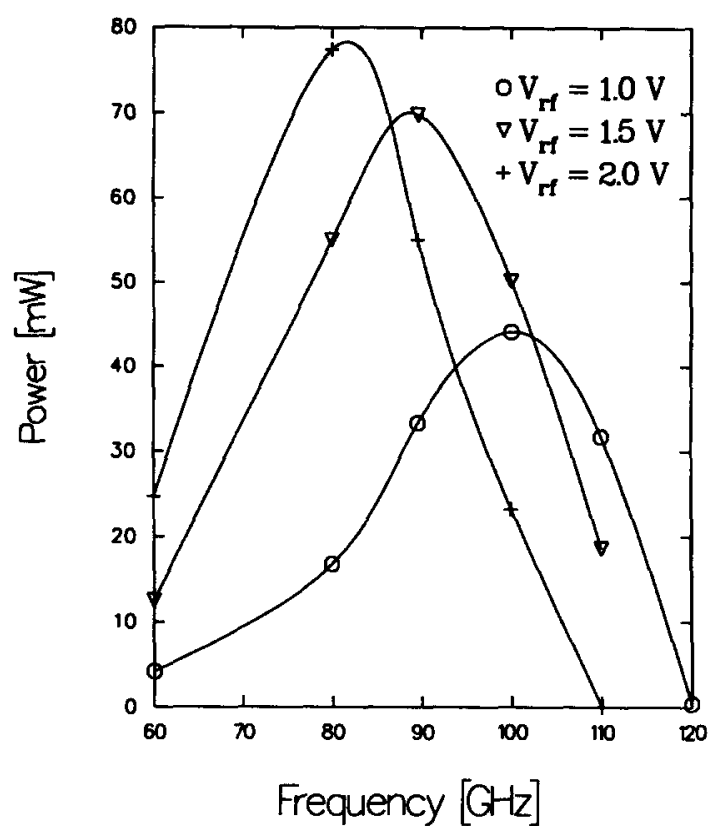

Fig. 6. Predicted output power as a function of frequency for three RF voltages $(1.0,1.5$ and $2.0 \mathrm{~V})$. The d.c. voltage is $4.0 \mathrm{~V}$ and the operating temperature is $450 \mathrm{~K}$.

device is set to $50 \mu \mathrm{m}$ which corresponds to the size of the tested diode. As a result, the load resistance of the resonant circuit is adjusted until the oscillation condition is satisfied. This procedure is repeated at various frequencies and RF voltages. Figure 4 shows the predicted conversion efficiency as a function of frequency for three RF voltages. At a given RF voltage, the efficiency curve exhibits a peak as a function of frequency. This peak occurs at $100 \mathrm{GHz}$ for an RF voltage of $1 \mathrm{~V}$ and has a value of $2 \%$. As the RF voltage amplitude is increased, the peak value increases and shifts to lower frequencies: $90 \mathrm{GHz}$ for an RF voltage of $1.5 \mathrm{~V}$ and $80 \mathrm{GHz}$ for an $\mathrm{RF}$ voltage of $2.0 \mathrm{~V}$. A peak is obtained because the efficiency is directly proportional to the RF voltage amplitude and the device equivalent negative conductance. The conductance decreases as the RF voltage is increased, therefore the product of the conductance and the RF voltage amplitude exhibits a maximum. The shift to lower frequencies could be explained as follows. Consider the effect of increasing the RF voltage from 1.0 to $2.0 \mathrm{~V}$. In the first half of the cycle, the voltage across the device extends further into the negative differential region. The average velocity and therefore the current decrease. With a lower average velocity, it takes a longer time for the space charge layer to cross the active region. Since the frequency is fixed, the current rise due to space charge should exhibit a delay compared with the $1.0 \mathrm{~V} \mathrm{RF}$ voltage case. This is indeed observed as illustrated in Fig. 5 where the current density is plotted at $105 \mathrm{GHz}$ for two RF voltages: 1.0 and $2.0 \mathrm{~V}$. The current peak is clearly shifted to the right (or delayed in time) as the RF voltage is increased from 1.0 to $2.0 \mathrm{~V}$. The simulation 
predicts oscillations in a wide-band of frequencies from 60 to $110 \mathrm{GHz}$. This is in agreement with the known behavior of Gunn devices as well as our experimental results. The corresponding predicted RF power is shown in Fig. 6. Similar to the efficiency curve, the power peaks at a particular frequency which shifts down as the RF voltage is increased. The model predicts up to $80 \mathrm{~mW}$ at $80 \mathrm{GHz}$ with an $\mathrm{RF}$ voltage of $2.0 \mathrm{~V}$.

It is now possible to compare the experimental with the theoretical results. Referring to Fig. 4 reveals that the predicted efficiency at $80 \mathrm{GHz}$ varies from $0.6 \%$ to more than $3 \%$ as the RF voltage is increased from 1.0 to $2.0 \mathrm{~V}$. Therefore there exists an intermediate RF voltage that yields an efficiency of $1.6 \%$. Similarly, inspection of the output power at $80 \mathrm{GHz}$ (Fig. 6) shows that power levels at $80 \mathrm{GHz}$ ranging from $15 \mathrm{~mW}$ to near $80 \mathrm{~mW}$ are predicted. The experimentally obtained power of $40 \mathrm{~mW}$ at $80 \mathrm{GHz}$ falls within this range. It is important to realize that higher power and efficiency levels could be predicted by increasing the d.c. voltage and changing the RF voltage. However such operation point might correspond to an excessive temperature rise or require unrealistic load impedance level. In the actual operation of the Gunn device, the resonant cavity determines the oscillation frequency according to the impedance it provides to the diode terminals.

In summary, an ensemble Monte Carlo model is developed for the simulation of InP Gunn devices. Choosing the material parameters needed by this model is not a trivial task. Typical material parameters taken from the literature were not capable of predicting the obtained experimental results. A more accurate set of parameters was obtained by comparing the theoretical results with a well characterized $W$. band InP Gunn device. It was found necessary to decrease the intervalley energy separation and increase the effective mass in the satellite valleys. This suggests that the InP material parameters are sensitive to the operating temperature and a characterization of this dependence is an interesting subject for further study.

\section{5. $1.0 \mu \mathrm{m}$ InP GUNN STRUCTURES}

In the previous section, a $1.7 \mu \mathrm{m}$ long InP Gunn structure was shown to oscillate at frequencies in the W-band. For higher fundamental frequencies, it is necessary to decrease the device length and increase the doping level in the active region. In this section, two $1 \mu \mathrm{m}$ structures with different doping profiles are considered.

\subsection{Flat doping profile}

A $1.0 \mu \mathrm{m} \mathrm{InP}$ structure has been designed with a flat doping profile. The wafer was grown by CBE (Chemical Beam Epitaxy). The doping in the active region was estimated to be $2.5 \times 10^{16} \mathrm{~cm}^{-3}$ from $C-V$ measurements. This doping is slightly higher than what is typically used for $1 \mu \mathrm{m}$ structures. The wafer

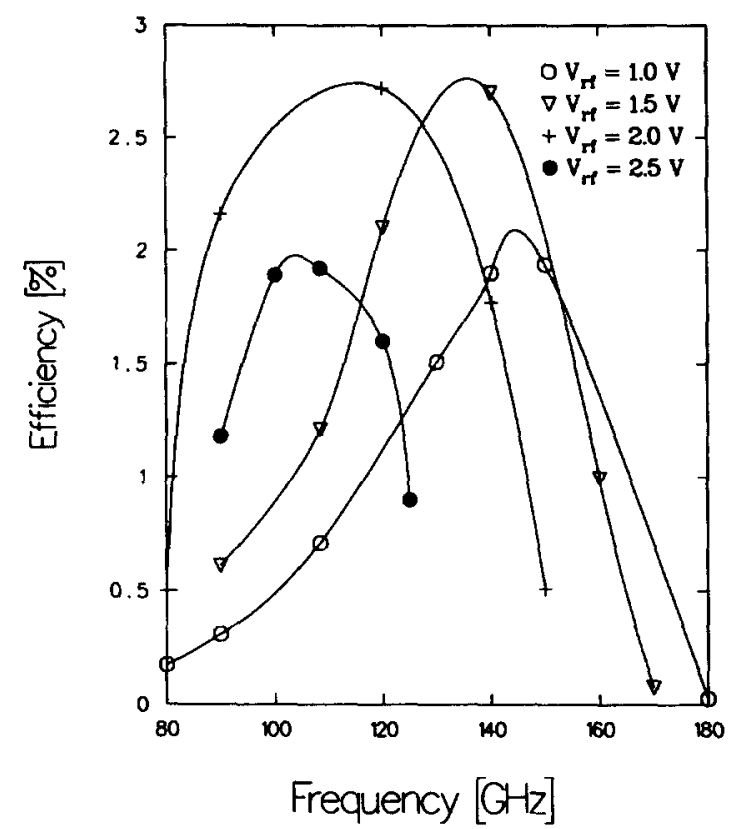

Fig. 7. Predicted efficiency as a function of frequency for a $1 \mu \mathrm{m}$ Gunn structure with flat doping profile. The d.c. voltage is $4.1 \mathrm{~V}$ and the operating temperature is $480 \mathrm{~K}$.

was processed and $35 \mu \mathrm{m}$ diodes were packaged and tested in a resonant cap waveguide cavity. Oscillations were obtained at $108.3 \mathrm{GHz}$ with $33 \mathrm{~mW} \mathrm{CW}$ output power and $1.87 \%$ efficiency. The diode was biased at $4.1 \mathrm{~V}$ and has a d.c. current of $430 \mathrm{~mA}$. The above result corresponds to the highest reported output power from an $n^{+}-n-n^{+}$structure, with a flat doping profile, at this frequency.

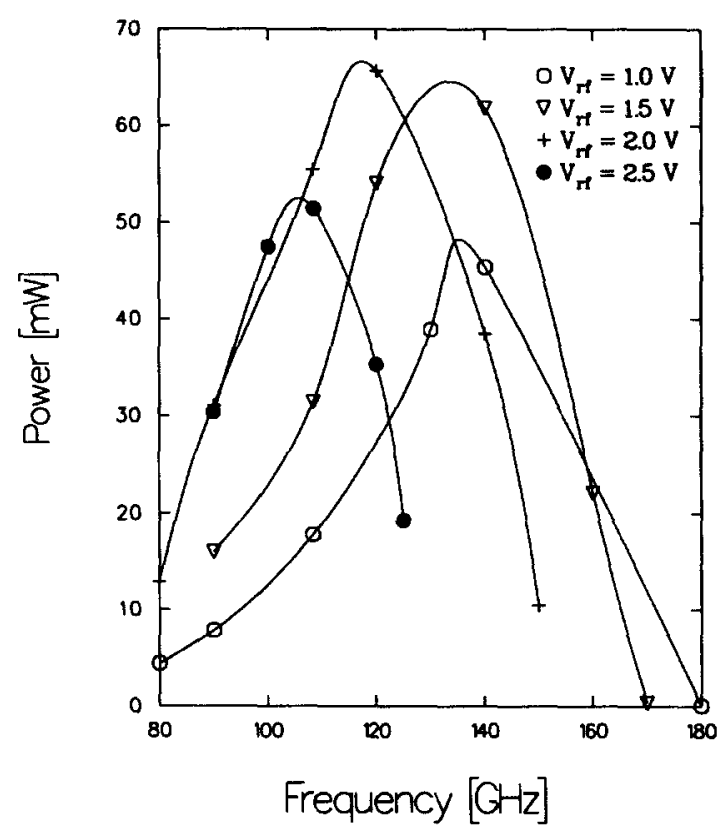

Fig. 8. Predicted output power as a function of frequency for a $1 \mu \mathrm{m}$ Gunn structure with flat doping profile. The d.c. voltage is $4.1 \mathrm{~V}$ and the operating temperature is $480 \mathrm{~K}$. 
A structure similar to the experimental device has been simulated. The bias voltage was set to $4.1 \mathrm{~V}$ and the operating temperature to $480 \mathrm{~K}$. The actual device operating temperature is estimated to be close to $500 \mathrm{~K}$ since most of the devices failed as the bias voltage is increased beyond 5 to $5.5 \mathrm{~V}$. The performance was evaluated as a function of frequency and for different $\mathrm{RF}$ voltages $(1.0,1.5,2.0$ and $2.5 \mathrm{~V})$. Figure 7 shows a plot of the predicted conversion efficiency as a function of frequency. At the lowest $R F$ voltage, the efficiency peaks around $145 \mathrm{GHz}$ and reaches a value of $2 \%$. As the RF voltage is increased, the frequency at which the peak occurs shifts down. At the same time the maximum efficiency initially increases, reaches a value close to $3 \%$ for an $\mathrm{RF}$ voltage between 1.5 and $2.0 \mathrm{~V}$, then decreases as the RF voltage is increased further.

The corresponding plot of the RF power vs frequency is shown in Fig. 8. More than $60 \mathrm{~mW}$ output power is predicted near $120 \mathrm{GHz}$. For an RF voltage of $2.5 \mathrm{~V}$, the power peaks near $108 \mathrm{GHz}$ and the oscillation bandwidth becomes smaller compared with lower RF voltages. The predicted power at $108.3 \mathrm{GHz}$ in this case is approximately $50 \mathrm{~mW}$. This value is in good agreement with the experimental results when taking into account cavity losses.

\subsection{Graded doping profile}

This section examines ways of enhancing the performance of InP Gunn devices in the D-band. One method consists of specifying a nonuniform doping profile in the active region and in particular, a linearly graded profile. In this case, the doping should increase from the cathode region toward the anode region, otherwise the performance is likely to be worsened[24]. The advantages of a linearly doping profile include reducing the peak electric field, lowering the current density, and improving the efficiency and output power. The peak electric field in a Gunn structure occurs near the anode region. With a linear doping profile, the peak is reduced because electrons diffuse from the high doped region near the anode to the low doped region at the cathode. The reduction in the field has two desired effects: first, a higher cathode field results in a larger fraction of the electrons transferring to the upper valleys over shorter distance, second a lower anode field allows the application of a larger d.c. bias without reaching breakdown. These effects improve the efficiency and the output power. A graded doping profile also results in a lower current density than would be obtained from a uniformly doped structure with similar doping level. This behavior is a consequence of the higher fraction of electrons in the upper valleys which reduces the average velocity.

Szubert et al. have compared the performance of W-band GaAs Gunn structures with flat and graded doping profiles[25]. The graded structures showed improved performance compared to flat doped structures with a similar $N \times L$ product, where $N$ repre- sents the doping and $L$ the length of the active region. Similarly, Kurita et al.[26] have reported on the performance of InP W-band Gunn devices with graded doping profiles. The best results obtained correspond to a $1.1 \mu \mathrm{m}$ structure with a linear doping profile increasing from $7.5 \times 10^{15}$ to $1.65 \times 10^{16} \mathrm{~cm}^{-3}$. The output power was $135 \mathrm{~mW}$ with $3 \%$ efficiency at $90 \mathrm{GHz}$ and $117 \mathrm{~mW}$ with $2.7 \%$ efficiency at $94 \mathrm{GHz}$. Although the devices were mounted on diamond heat sinks, higher power levels have been reported using flat doped structures [2]. As indicated earlier, the emphasis in our effort is to develop InP fundamental oscillators for D-Band operation. Therefore, our structure has been designed with a shorter active region and higher doping gradient compared to the structures investigated by Kurita $e t$ al.[26].

A wafer with a graded doping profile was designed and grown by MOCVD. The structure has a $1 \mu \mathrm{m}$ active region with a doping linearly increasing from $7.5 \times 10^{15} \mathrm{~cm}^{-3}$ at the cathode side of the active region to $2.0 \times 10^{16} \mathrm{~cm}^{-3}$ at the anode side. Samples were processed with integrated heat sinks consisting of plated gold and silver layers. $45 \mu \mathrm{m}$ diodes were mounted on copper heat sinks and tested in a D-band waveguide cavity. Oscillations were obtained at $120 \mathrm{GHz}$ with a power of $20 \mathrm{~mW}$ and at $136 \mathrm{GHz}$ with a power of $10 \mathrm{~mW}$. These devices are believed to operate in the fundamental mode since the measured $Q$ values were between 30 and 100 around $120 \mathrm{GHz}$ using a self-injection locking method. These $Q$ values are not a characteristic of a harmonic mode operation $[27,28]$. Even though these results are preliminary, they represent the best performance from Gunn Devices reported at these frequencies.

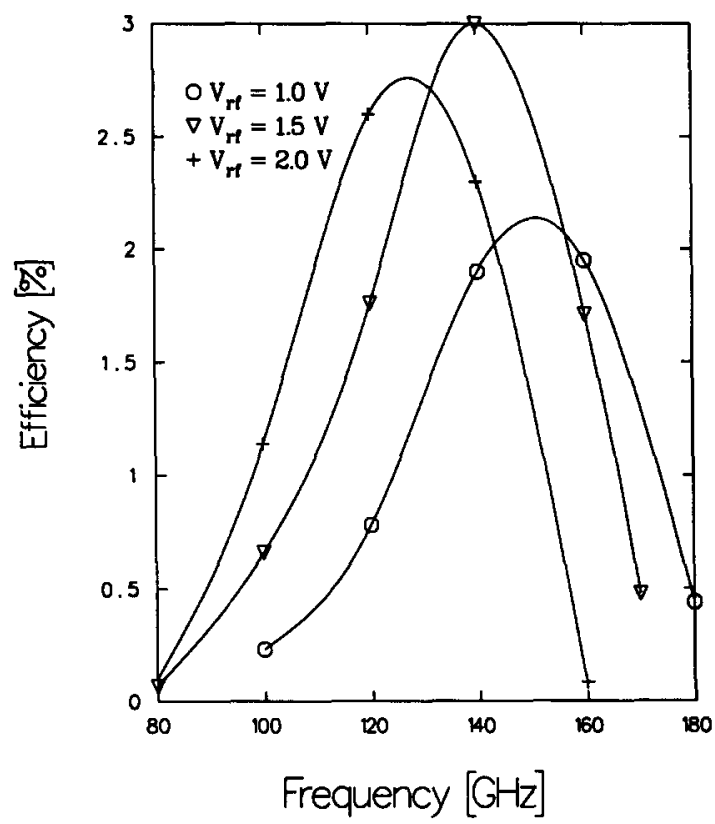

Fig. 9. Predicted efficiency as a function of frequency for a $1 \mu \mathrm{m}$ Gunn structure with graded doping profile. The d.c. voltage is $4.1 \mathrm{~V}$ and the operating temperature is $400 \mathrm{~K}$. 


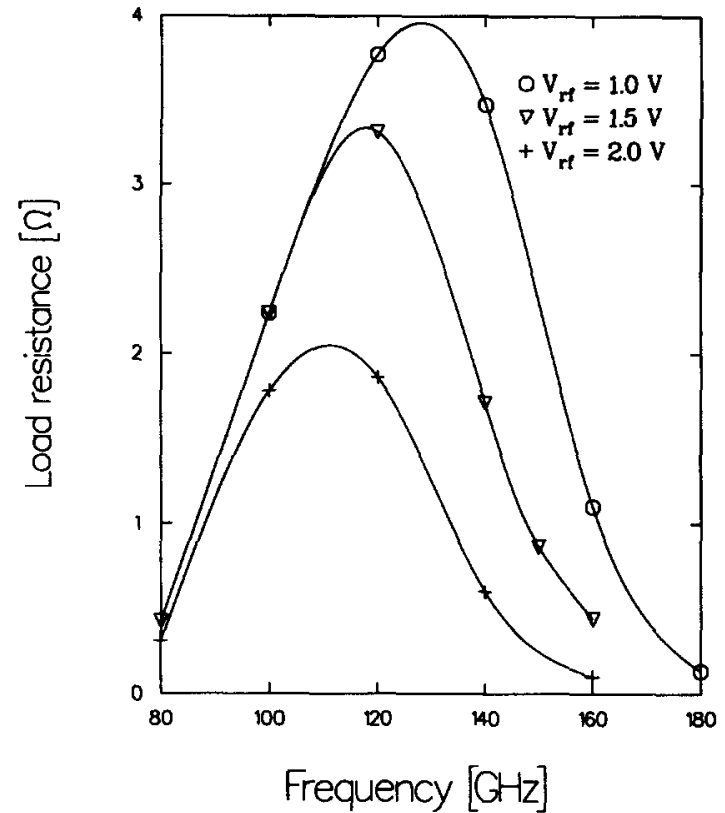

Fig. 10. Variation of the load resistance as a function of frequency for a $1 \mu \mathrm{m}$ Gunn structure with graded doping profile. The d.c. voltage is $4.1 \mathrm{~V}$ and the operating temperature is $400 \mathrm{~K}$.

Simulations were carried out on a similar structure using the Monte Carlo model. The d.c. bias voltage was set to $4.1 \mathrm{~V}$, the operating temperature at $400 \mathrm{~K}$, and the device diameter was fixed at $45 \mu \mathrm{m}$. Figure 9 shows the predicted efficiency as a function of frequency at various RF voltages. The oscillations occur at frequencies ranging from 80 to $180 \mathrm{GHz}$, however

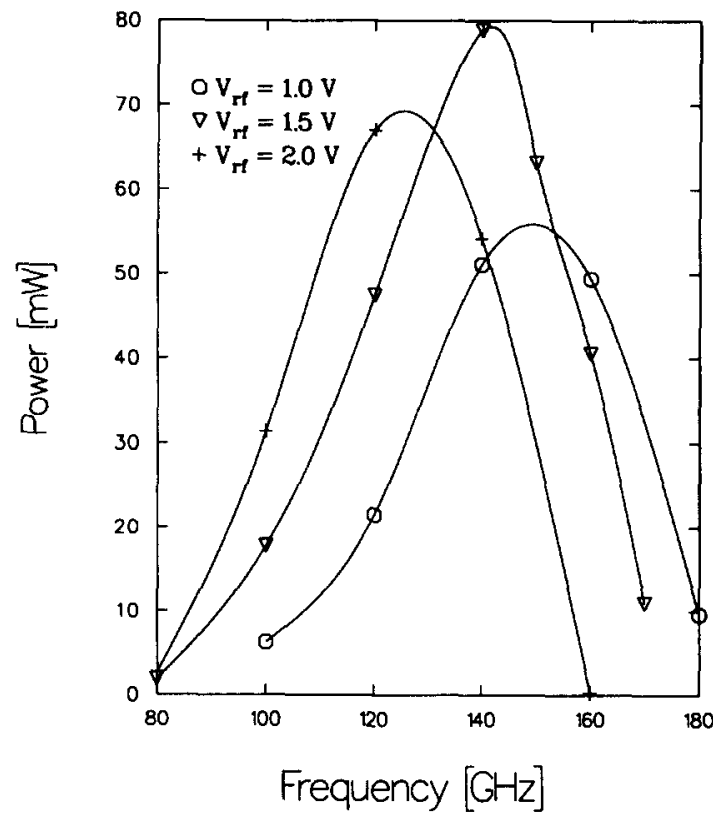

Fig. 11. Predicted output power as a function of frequency for a $1 \mu \mathrm{m}$ Gunn structure with graded doping profile. The d.c. voltage is $4.1 \mathrm{~V}$ and the operating temperature is $400 \mathrm{~K}$. at the two extremes the oscillation condition requires load resistances less than $1 \Omega$. This is illustrated in Fig. 10 which shows the load resistance as a function of frequency for different RF voltages. The best efficiency occurs near $140 \mathrm{GHz}$ and reaches a value of $3 \%$. The output power also peaks near $140 \mathrm{GHz}$ as shown in Fig. 11 where $80 \mathrm{~mW}$ is predicted for an RF voltage of $1.5 \mathrm{~V}$. The simulation predicts oscillations in a frequency range corresponding to what has been observed experimentally, but with better performance. As mentioned above, the experimental results are preliminary and more work needs to be done to further improve the performance of the graded structures in the D-band.

\section{CONCLUSIONS}

A method has been developed for estimating the material parameters used in the Monte Carlo model. By comparing simulation and experimental results at high frequencies, more accurate material parameters were obtained. It was found necessary to use low values for the intervalley energy separation and high values for the deformation potentials than normally used in the literature. A possible reason for these trends is the high operating temperature of the Gunn device.

Simulation results have shown that it is possible to operate fundamental mode InP Gunn devices in the D-band frequency region. The operation requires structures with near micron dimensions. Two such structures have been designed, modeled, fabricated, and tested. The first structure consisted of a $1 \mu \mathrm{m}$ active region uniformly doped at $2.5 \times 10^{16} \mathrm{~cm}^{3}$. The second structure had a linearly graded doping profile increasing from $7.5 \times 10^{15} \mathrm{~cm}^{-3}$ at the cathode side to $2.0 \times 10^{16} \mathrm{~cm}^{-3}$ at the anode side. An improved processing technology for high frequency Gunn diode operation was developed. Using this technology, preliminary results were very encouraging and represent the state of the art at these frequencies. Both structures operated over roughly the same frequency range, however the graded structure yielded better performance at the high frequency end. This observation confirms the claim that nonuniform doping profiles are superior to flat doping profiles in terms of performance and high frequency capabilities.

The theoretical simulations with the improved material parameters predict even higher power levels at these frequencies. It is the opinion of the authors that better experimental results could be achieved around $140 \mathrm{GHz}$. This requires optimizing the doping profile, reducing further the contact resistances, and developing better heat sinks and packaging techniques.

Acknowledgements - The authors would like to thank G. Munns and Dr M. Sherwin for growing the InP wafers with the flat doping profile and Yoshio Saito at TRW for providing the quartz rings. This work was supported by the Center for Space Terahertz Technology under Contract No. NAGW-1334. 


\section{REFERENCES}

I. S. J. J. Teng and R. E. Goldwasser, IEEE Electron Device Lett. 10, 412 (1989).

2. M. A. di Forte-Poisson, C. Brylinski, N. Proust and D. Pons, SPIE 1144, 551 (1989).

3. I. G. Eddison, I. Davies, P. L. Giles and D. M. Brookbanks, Electron. Lett. 17, 758 (1981).

4. L. Wandinger, Microwave J., p. 71 (1981).

5. M. A. di Forte-Poisson, C. Brylinski, G. Colomer, D. Osselin, S. Hersee, J. P. Duchemin, F. Azan, D. Lechevallier and J. Lacombe, Electron. Lett. 20, 1061 (1984).

6. B. Fank, J. Crowley, D. Tringali and L. Wandinger, SPIE 1144, 534 (1989).

7. Anders Rydberg, IEEE Electron Device Lett. 11, 439 (1990).

8. R. T. Gallagher, Electronics Week, p. 25, Jan 14 (1985).

9. B. Fank, Microwave J., p. 95 (1984).

10. W. H. Haydl, Electron. Lett. 17, 825 (1981).

11. S. M. Sze, High-Speed Semiconductor Devices Physics, p. 571. Wiley-Interscience, New York (1990).

12. B. Bayraktaroglu and H. D. Shih, Electron. Lett. 22, 562 (1986).

13. H. Eisele, Solid-St. Electron. 32, 253 (1989).

14. J. M. Woodall, J. L. Freeouf, G. D. Pettit, T. Jackson and P. Kirchner, J. Vac. Sci. Technol. 19, 626 (1981).
15. W. Fawcett, A. D. Boardman and S. Swain, J. Phys. Chem. Solids 30, 643 (1969).

16. R. A. Zettler and A. M. Cowley, Electron. Lett. 5, 693 (1969).

17. S. Y. Narayan and J. P. Paczkowski, RCA Rev. 33, 752 (1972).

18. A. Paolella, R. L. Ross and J. Ondria, Microwave J., p. 149 (1986)

19. K. Brennan, K. Hess, J. Y. Tang and G. J. Iafrate, IEEE Trans. Electron Devices ED-30, 1750 (1983).

20. D. C. Herbert, W. Fawcett and C. Hilsum, J. Phys. C: Solid State Phys. 9, 3969 (1976).

21. G. H. Glover, J. appl. Phys. 44, 1295 (1973).

22. T. J. Maloney and J. Frey, J. appl. Phys. 48, 781 (1977).

23. M. V. Fischetti, IEEE Trans. Electron Devices ED-38, 634 (1991).

24. Miichihisa Suga and Kenji Sekido, IEEE Trans, Electron Devices ED-17, 275 (1970).

25. J. M. Szubert, J. Barstow, R. B. Beall and J. J. Harris, Solid-St. Electron. 33, 10355 (1990).

26. H. Kurita, A. Yokohata, A. Kodama, K. Suga, S. Kato and M. Ohmori, Proc. Third Int. Conf. on Indium Phosphide and Related Materials, p. 280 (1991).

27. H. Barth, IEEE MTT-S Dig., p. 179 (1986)

28. I. G. Eddison and D. M. Brookbanks, Electron. Lett. 17, 112 (1981). 